\title{
Method of Squaring Any Number Retaining All the Digits in the Result and Use of Positive and Negative Digits in A Number
}

\author{
Dr. A. M. Chandra \\ (Department of Civil Engineering, Sharda University, India)
}

\begin{abstract}
With the development of digital electronic computers and calculators the mathematical operations have become easier and faster but there is always a limitation of retaining the number of digits in the results of the operations. One has to see the retained number of digits in the result produce how much error in the other quantities which are computed using them. But it is a fun to retain all the digits in the result of square of a number of any sizes. Sometimes all the digits in the result may be required for some accurate scientific investigations when computers fail to provide the same. The author has developed this method of squaring any number retaining all the digits in the result.
\end{abstract}

\section{Introduction}

The method of squaring a number having any number of digits, and retaining all the digits in the result came to mind of the author while going through the methods of basic mathematical operations using ancient Indian Vedic mathematics. In this paper the author has tried to explain all the steps as far as possible and finally a number of sixteen digits have been squared showing that the method is simpler for such cases, and that all 32 digits in the result have been retained. This paper also explains that how a number containing large digits (between 6 to 9) can be converted into a number containing small digits (between 0 to 5) which makes the computations easier and faster. Such converted equivalent numbers contain positive and negative digits which is not in the present employed systems of mathematics. A method of checking the correctness of the results has also been presented in this paper.

\section{New Method of Squaring a Number}

The existing method used for squaring a number is to write the number twice and multiply them to get the square of the number. In the new method it is not required to write the number to be squared twice. The number is written, and the operations start from the right most digit and proceed towards left leaving the digits one by one. The basic principle states that

"Square the right most digit, say $a$, and then multiply the remaining digits on the left side by twice of a." This process has to be done in similar manner for the remaining digits proceeding towards left starting from the right most digit of each remaining digits of the number.

Taking an example of a number representing its digits as $a, b, c$, and $d$, let the number be $d c b a$. To get its square by the rule given above, the step by step procedure is given below:

$\begin{array}{lcr} & \text { The number is } d c b a & a^{2}=A \\ \text { Step }-1 & \text { Write square of } a & (2 a) \square \square \square d c b \square \square=B \\ \text { Step }-2 & \text { Multiply the remaining digits } d c b \text { by } 2 a & b^{2} \square=C \\ \text { Step }-3 & \text { Write square of } b & (2 b) \square \square \square d c \square \square=D \\ \text { Step }-4 & \text { Multiply the remaining digits } d c \text { by } 2 b & c^{2} \square=E \\ \text { Step }-5 & \text { Write square of } c & (2 c) \square \square \square d \square \square \\ \text { Step }-6 & \text { Multiply the remaining digit } d \text { by } 2 c & d^{2}=G\end{array}$

To get the final answer one has to see that at which place the results of the above steps are to be placed. If the places of the digits in a number are then

Step -1 result $A$ at place 1

Step -2 result $B$ at place 2

Step -3 result $C$ at place 3

Step -4 result $D$ at place 4

Step -5 result $E$ at place 5

Step -6 result $F$ at place 6

Step -7 result $G$ at place 7

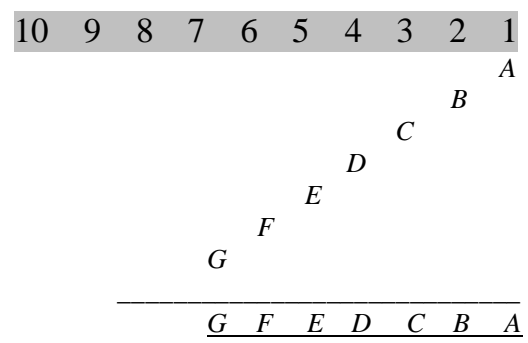

Final answer (adding the digits vertically as we do summation)

\begin{tabular}{lllllll}
$G$ & $F$ & $E$ & $D$ & $C$ & $B$ & $A$ \\
\hline
\end{tabular}


Method of Squaring Any Number Retaining All the Digits in the Result and Use of Positive and

\begin{tabular}{clc}
\hline Let us find out square of & 23051 & \\
Step -1 & Write square of $1 \quad 1^{2}$ & 4 \\
Step -2 & Multiply the remaining digits 2305 by $2 \square \square 1$ & 25 \\
Step -3 & Write square of $5 \quad 5^{2}$ & 2300 \\
Step -4 & Multiply the remaining digits 230 by $2 \square \square 5$ & 0 \\
Step -5 & Write square of $0 \quad 0^{2}$ & 00 \\
Step -6 & Multiply the remaining digit 23 by $2 \square \square 0$ & 9 \\
Step -7 & Write square of $3 \quad 3^{2}$ & 12 \\
Step -8 & Multiply the remaining digit 2 by $2 \square \square 3$ & 4 \\
Step -9 & Write square of 2 $\quad 2^{2}$ &
\end{tabular}

To get the final answer one has to see that at which place the results of the above steps are to be placed. If the places of the digits in a number are then

Step -1 result at place 1

Step -2 result at place 2

Step -3 result at place 3

Step -4 result at place 4

Step -5 result at place 5

Step -6 result at place 6

Step -7 result at place 7

Step -8 result at place 8

Step -9 result at place 9

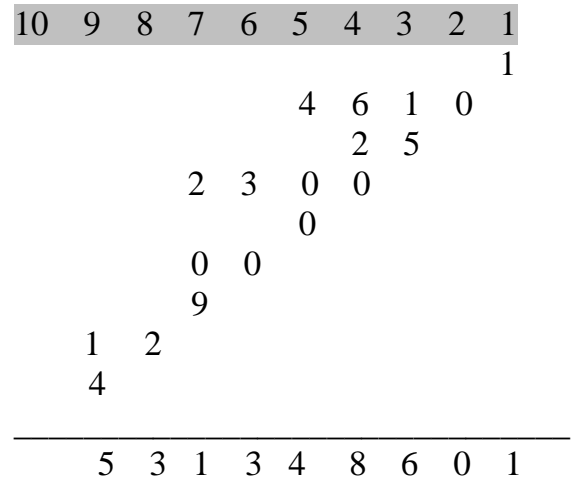

Final answer

Now let us take another number, and do all the above steps as below:

The number is

$$
\begin{aligned}
& \text { Step }-1 \\
& \text { Step }-2 \\
& \text { Step }-3 \\
& \text { Step }-4 \\
& \text { Step }-5 \\
& \text { Step }-6 \\
& \text { Step }-7 \\
& \text { Step }-8 \\
& \text { Step }-9 \\
& \text { Step }-10 \\
& \text { Step }-11
\end{aligned}
$$

Final answer
231243

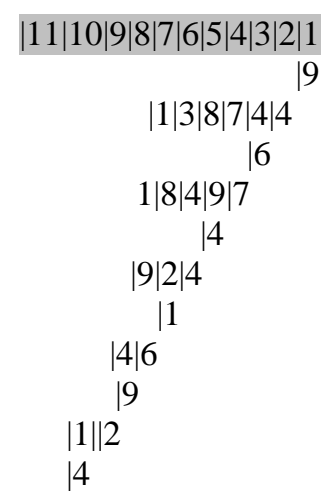

$|5| 3|4| 7|3| 3|2| 5|0| 4 \mid 9$

\section{Breaking the Number in Parts to get Square of a Large Number}

If a number consists of, say 16 digits, and in the result of its square all the digits are to be retained, it is going to occupy large horizontal and vertical space. To avoid such a situation, the above operations of squaring can be performed in parts breaking the number in parts, and then adding the results of all the parts systematically. The method is explained in the following example:

Let the number be 6321501191232103, and it is divided in four parts as

$$
6321 \square 5011 \square 9123 \square 2103
$$

Step -1 Consider all the digits and the right most part.

Step -2 Consider all the digits of the remaining three parts and its right most part.

Step -3 Consider all the digits of the remaining two parts and its right most part.

Step -4 Consider all the digits of the remaining one part.

Step -5 Add the results systematically.

To get the square of 6321501191232103, follow the above steps as below:

Step -1 Consider all the digits and the right most part.

$$
6321 \square 5011 \square 9123 \square 2103
$$


Apply the operations explained in Sec. 2.

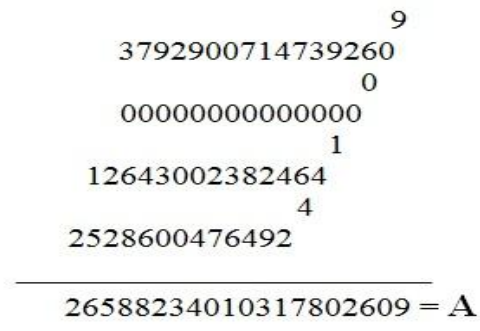

Step - 2 Consider all the digits of the remaining three parts and its right most part.

$$
6321 \square 5011 \square \mathbf{9 1 2 3}
$$

Apply the operations explained in Sec. 2.

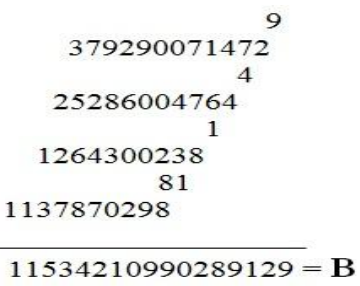

Step - 3 Consider all the digits of the remaining two parts and its right most part.

$$
6321 \square \mathbf{5 0 1 1}
$$

Apply the operations explained in Sec. 2.

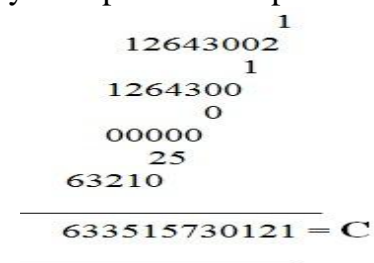

Step -4 Consider all the digits of the remaining one part.

6321

Apply the operations explained in Sec. 2.

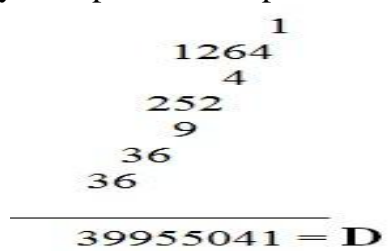

To get the final answer, add the results $\mathbf{A}, \mathbf{B}, \mathbf{C}$, and $\mathbf{D}$, systematically. If the number has been divided taking two digits, below $\mathbf{A}$ write $\mathbf{B}$ leaving $2 \square \square 2=4$ digits from the left, and similarlyC, and $\mathbf{D}$. If divided taking four digits, below A write B leaving $2 \square \square 4=8$ digits from the left, and similarlyC, and D. Thus

$$
\begin{aligned}
& 26588234010317802609=\mathbf{A} \\
& 11534210990289129=\mathbf{B} \\
& 633515730121=\mathbf{C} \\
& 39955041=\mathbf{D}
\end{aligned}
$$

Add the numbers vertically to get the final result $=\quad \mathbf{3 9 9 6 1 3 7 7 3 1 0 7 4 8 8 9 7 2 6 2 9 2 3 2 1 7 8 0 2 6 0 9}$

\section{Checking the correctness of the results}

We have a very easy method of checking the results. In this method sum of all the digits of a number is brought to a single digit. To check the result of square of a number, take sum of all the digits of the number 
making it a single digit, square it, and again find the sum of all the digits making it to a single digit. This single digit number will be same as the sum of all the digits of the result brought to a single digit.

Let us check the results of all the examples discussed above.

In the first example, the sum of the digits of 23051 is $(2+3+0+5+1)=(11) \square(1+1)=2$. The square of 2 is 4 which is a single digit in itself. The sum of all the digits of the result 531348601 is $(5+3+1+3+4+8+6+0+1)=$ (31) $\square(3+1)=4$. As both are same the result is correct.

In the second example the number is 231243 .

For the number $\quad(2+3+1+2+4+3)^{2}=(15)^{2} \square \square\left(1+5^{2}=(6)^{2}=(36) \square \square(3+6)=9\right.$

For the result $\quad(5+3+4+7+3+3+2+5+0+4+9)=(45) \square(4+5)=9$ (Okay)

In the third example the number is 6321501191232103.

For the number $\quad(6+3+2+1+5+0+1+1+9+1+2+3+2+1+0+3)^{2}=(4)^{2}=(16) \square \square(1+6)=7$

For the result $\quad(3+9+9+6+1+3+7+7+3+1+0+7+4+8+8+9+7+2+6+2+9+2+3+2+1+7+8+0+2+6+0+9)=$ (151) $\square(1+5+1)=7$ (Okay)

\section{Use of Positive and Negative Digits in a Number}

In the ancient Indian Vadic mathematics the numbers can have positive and negative digits contrary to either positive or negative in the present system in use. This system, therefore, makes the long manual calculations easier and faster as the digits greater than 5 can be written as equivalent digits smaller than 5 .

The principle of positive and negative digits in a number is explained below. The negative digit is indicated in bold italic number, e.g. $\square \square$ as 4 .

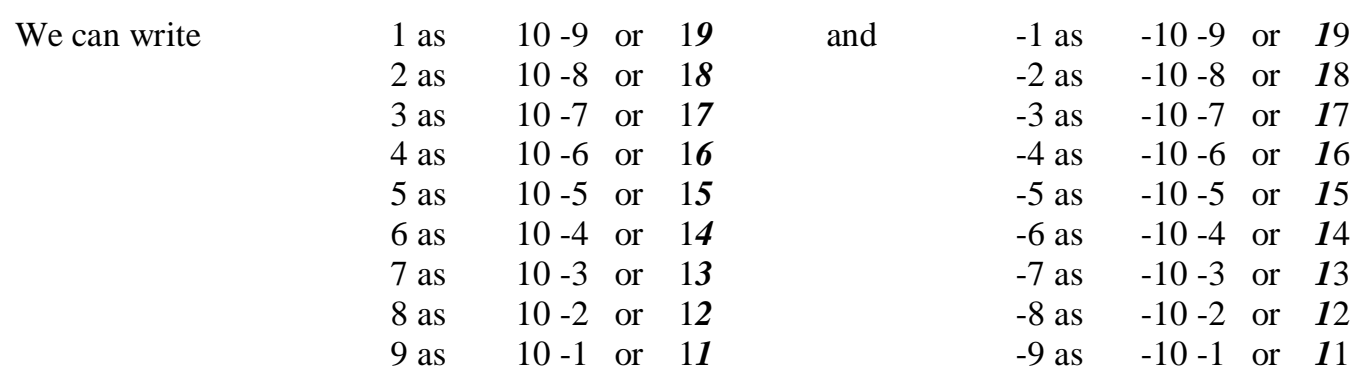

Now some examples are taken below to explain how the numbers can be written in positive and negative digits avoiding digits greater than 5 .

Example: 1 The number 17 can be written as $10=10$

$$
+7 \quad+13=23
$$

Example: 2 The number 59 can be written as $\quad 50=50$

$$
+9 \quad+11=61
$$

But 6 is greater than 5 , therefore write 14 for 6 .

Thus $\quad 61$ becomes 141 or $59=141$

Example: 2 the number 87969 can be written in similar manner as 7000

$$
\begin{array}{r}
80000=120000 \\
13000 \\
1100
\end{array}
$$

$60-140$

$9 \quad 11$

Adding algebraically vertically in each column, we get

$87969 \quad 112031$

It can be seen that 87969 which has digits greater than 5 has been converted into an equivalent number 112031 which has all the digits less than 5. All mathematical operations using a number consisting of positive and negative digits become easier and faster. Now we will find out the square of the 87969 using this directly and also using the equivalent number, and will compare the two processes to see that using the equivalent number is easier and faster if one does himself mentally.
$(87969)^{2}$
1583361
$\left(\begin{array}{llllllllll}1 & 1 & 2 & 0 & 3 & 1\end{array}\right)^{2}$
224061
105516
67209
15741
0000
1169
444 


\begin{tabular}{ll}
\hline 64 & 21 \\
\hline $7738544961=\mathbf{A}$ & $\frac{1}{12342544961=} \mathbf{B}$
\end{tabular}

In the above two answers $\mathbf{A}$ and $\mathbf{B}$, we find that $\mathbf{A}$ has all positive digits whereas $\mathbf{B}$ has positive and negative digits both. Mathematically $\mathbf{B}$ is acceptable but we are used to the numbers having all the digits either positive or negative, and this can be obtained easily using the concept of converting the number in positive and negative digits explained above. Thus

\begin{tabular}{|c|c|c|}
\hline 12000000000 & $=$ & 8000000000 \\
\hline $\begin{array}{lllllllll}3 & 0 & 0 & 0 & 0 & 0 & 0 & 0 & 0\end{array}$ & & 1700000000 \\
\hline 4000000000 & & 40000000 \\
\hline 200000000 & & 18000000 \\
\hline 504000 & & 504000 \\
\hline 400000 & & 400000 \\
\hline 4000 & & 4000 \\
\hline 900 & & 900 \\
\hline 60 & & 60 \\
\hline 1 & & 1 \\
\hline 2342544 & & 7738544961 \\
\hline
\end{tabular}

Let us check the result.

For the number $(8+7+9+6+9)^{2}=(3)^{2}=9$

For the result $-1 \quad(7+7+3+8+5+4+4+9+6+1)=(54) \square(5+4)=9$ (Okay)

For the result $-2 \quad(1 \square 2 \square 3+4 \square 2+5+4+4++9+6+1) \neq 18) \square(1+8)=9$ (Okay)

One has to remember that the process shown above of converting a number containing positive and negative digits into an equivalent number containing positive (or negative) digits, is to be done mentally.

\section{Conclusions}

This paper clearly illustrates a new method of squaring any number retaining all the digits in the result. The steps for squaring are to be done mentally and the result of each step is to be written one after the other at proper places. As the developed method is faster compared to the traditional method when working with numbers having large number of digit, it can be used in electronic computers by developing a suitable algorithm to make the computations still faster. Further, the method becomes more easy and fast when large digits are converted into equivalent smaller positive and negative digits in the number.

\section{Acknowledgements}

The author is indebted to all those teachers who gave lectures on Vadic mathematics about 20 years back for a course organized for the school children, and which was attended by the author as Professor along with the children. This course inspired him to work on several problems using the Sutras (rules) of Vedic mathematics, and during working on several problems the method of squaring any number was developed. 